\title{
Liouville Dynamics and the Conservation of Classical Information
}

\author{
A. R. Plastino* \\ Physics Department, University of Pretoria, Pretoria 0002, South Africa, and Faculty of Astronomy and Geophysics, La Plata \\ National University-CONICET, Casilla de Correo 727, La Plata 1900, Argentina
}

A. Daffertshofer ${ }^{\dagger}$

Faculty of Human Movement Sciences, Vrije Universiteit, van der Boechorststraat 9, 1081 BT, Amsterdam, The Netherlands

(Received 2 May 2004; published 20 September 2004)

\begin{abstract}
We show that the invariance of entropic distances under Liouville dynamics yields classical analogues of information-related, quantum mechanical impossible operations. By recourse to Fisher's information measure, we also establish a connection between such classically forbidden operations and statistical estimation theory.
\end{abstract}

DOI: 10.1103/PhysRevLett.93.138701

PACS numbers: 89.70.+c, 03.67.-a, 05.20.-y

The physics of information has attracted attention for more than half a century, though the number of studies in this field has been growing only in the last two decades or so [1-14]. Information-related concepts are considered important for various physical problems ranging from quantum mechanical aspects of the second law of thermodynamics [11] to the evolution of inhomogeneous cosmological models [12]. Recent investigations on novel and counter-intuitive ways of processing and transmitting (quantum) information [13,14] boosted interest in the physics of information across scientific communities.

Some fundamental aspects of quantum physics that are relevant for the processing of information admit to classical counterparts. Classical analogues of entanglement [15] and quantum search algorithms [16] as well as classical dynamical settings leading to non-Boolean logics $[17,18]$ have been identified. Lately, it has been shown [19] that Liouville dynamics complies with a classical counterpart of the celebrated quantum noncloning theorem $[20,21]$. Besides quantum cloning, however, there are other important examples of information-related processes forbidden by the laws of quantum mechanics, e.g., quantum deleting [22] and quantum disentangling [23]. The physical impossibility of those quantum operations has profound implications for both quantum information theory and quantum physics in general, being nowadays the focus of intensive research [24-27].

The aim of this Letter is to advance a general framework for the discussion of classical counterparts of some of the aforementioned quantum impossible processes. We focus on classical analogues of the noncloning and the nodeleting theorems, which we derive from the Liouville dynamics that governs the evolution of statistical ensembles. We will prove that the concomitant classically forbidden operations are not compatible with a profound property of Liouville dynamics: the conservation of the (Kullback-Leibler [28] and related) information distances. Exploring classical analogues of the alluded to theorems can help to distinguish between their essentially quantum mechanical features and aspects that may arise within purely classical probabilistic settings. This line of inquiry may contribute to a deeper understanding of the relationship between classical and quantum mechanics.

We study general classical deterministic dynamical systems, whose evolutions are governed by the equations of motion

$$
\frac{d \boldsymbol{x}}{d t}=\boldsymbol{v}(\boldsymbol{x}), \quad \text { with } \boldsymbol{x}, \boldsymbol{v} \in R^{N}
$$

where $\boldsymbol{x}$ denotes a point in the concomitant $N$-dimensional phase space [29]. The dynamics of a statistical ensemble of such systems can be described by a time-dependent probability distribution $\mathcal{P}(\boldsymbol{x}, t)$. Its dynamics is given by the Liouville equation,

$$
\frac{\partial}{\partial t} \mathcal{P}+\nabla(\boldsymbol{v} \mathcal{P})=0 .
$$

Considering two probability distributions, $\mathcal{P}_{1}$ and $\mathcal{P}_{2}$, we can define the integral

$$
G\left(\mathcal{P}_{1}, \mathcal{P}_{2}\right)=\int d x \mathcal{P}_{1} g\left[\frac{\mathcal{P}_{1}}{\mathcal{P}_{2}}\right],
$$

in which $g[\cdots]$ denotes an arbitrary function. Dependent on the explicit choice of the function $g[\cdots]$ this general integral provides a convenient way to measure timeinvariant relations (distances) between $\mathcal{P}_{1}$ and $\mathcal{P}_{2}$. Substituting (2) in the temporal derivative of (3) one finds through integration by parts 


$$
\begin{aligned}
\frac{d G}{d t} & =\int d \boldsymbol{x}\left[\frac{d \mathcal{P}_{1}}{d t}\left(g+\frac{\mathcal{P}_{1}}{\mathcal{P}_{2}} g^{\prime}\right)-\frac{d \mathcal{P}_{2}}{d t} \frac{\mathcal{P}_{1}^{2}}{\mathcal{P}_{2}^{2}} g^{\prime}\right] \stackrel{(2)}{=}-\int d x\left[\nabla\left(\boldsymbol{v} \mathcal{P}_{1}\right)\left(g+\frac{\mathcal{P}_{1}}{\mathcal{P}_{2}} g^{\prime}\right)-\nabla\left(\boldsymbol{v} \mathcal{P}_{2}\right) \frac{\mathcal{P}_{1}^{2}}{\mathcal{P}_{2}^{2}} g^{\prime}\right] \\
& =\int d \boldsymbol{x}\left[\boldsymbol{v}\left\{\mathcal{P}_{1} \nabla\left(g+\frac{\mathcal{P}_{1}^{2}}{\mathcal{P}_{2}^{2}} g^{\prime}\right)-\mathcal{P}_{2} \nabla\left(\frac{\mathcal{P}_{1}^{2}}{\mathcal{P}_{2}^{2}} g^{\prime}\right)\right\}\right] \\
& =\int d \boldsymbol{x}\left[\boldsymbol{v}\left\{\mathcal{P}_{1}\left[2 g^{\prime} \nabla\left(\frac{\mathcal{P}_{1}}{\mathcal{P}_{2}}\right)+\frac{\mathcal{P}_{1}}{\mathcal{P}_{2}} \nabla g^{\prime}\right]+-\mathcal{P}_{2}\left[g^{\prime} \nabla\left(\frac{\mathcal{P}_{1}^{2}}{\mathcal{P}_{2}^{2}}\right)+\frac{\mathcal{P}_{1}^{2}}{\mathcal{P}_{2}^{2}} \nabla g^{\prime}\right]\right\}\right]-\left[\overline{\boldsymbol{v}} \mathcal{P}_{1} g \mid=0 ;\right.
\end{aligned}
$$

here we assume that eventual surface terms vanish; $\overline{\mathbf{v}}$ denotes the sum of the components of $\boldsymbol{v}$. In order to illustrate this form, notice that the special case

$$
g\left[\frac{\mathcal{P}_{1}}{\mathcal{P}_{2}}\right]=\ln \frac{\mathcal{P}_{1}}{\mathcal{P}_{2}} \stackrel{G \rightarrow K}{\longrightarrow} K\left(\mathcal{P}_{1}, \mathcal{P}_{2}\right)=\int d x \mathcal{P}_{1} \ln \frac{\mathcal{P}_{1}}{\mathcal{P}_{2}}
$$

describes the Kullback-Leibler distance and that

$$
g\left[\frac{\mathcal{P}_{1}}{\mathcal{P}_{2}}\right]=\sqrt{\frac{\mathcal{P}_{2}}{\mathcal{P}_{1}} \rightarrow O} O\left(\mathcal{P}_{1}, \mathcal{P}_{2}\right)=\int d x \sqrt{\mathcal{P}_{1} \mathcal{P}_{2}}
$$

defines the overlap between $\mathcal{P}_{1}$ and $\mathcal{P}_{2}$. This overlap is closely related to a frequently used statistical distance between probability distributions [30] given by $\cos ^{-1}\left\{O\left(\mathcal{P}_{1}, \mathcal{P}_{2}\right)\right\}$. Suppose we have a composite system constituted by two statistically independent subsystems $a$ and $b$ described by a factorized joint probability distribution $\mathcal{P}=\mathcal{P}^{(a)} \mathcal{P}^{(b)}$. It follows from (5) that the Kullback distance between two such distributions verifies

$$
K\left(\mathcal{P}_{1}, \mathcal{P}_{2}\right)=K\left(\mathcal{P}_{1}^{(a)}, \mathcal{P}_{2}^{(a)}\right)+K\left(\mathcal{P}_{1}^{(b)}, \mathcal{P}_{2}^{(b)}\right) .
$$

That is, for factorized probability distributions the total distance becomes the sum of the individual Kullback distances between the two subsystems. In contrast, from (6) one obtains

$$
O\left(\mathcal{P}_{1}, \mathcal{P}_{2}\right)=O\left(\mathcal{P}_{1}^{(a)}, \mathcal{P}_{2}^{(a)}\right) O\left(\mathcal{P}_{1}^{(b)}, \mathcal{P}_{2}^{(b)}\right) .
$$

Utilizing the forms (5) and (6), we first turn our attention to the cloning process. For this sake, we assume two states $j=1,2$ of a tripartite system composed of a machine $(m)$, a source system $(s)$, and a target system $(t)$. In consequence, the initial states read

$$
\mathcal{P}_{j}=\mathcal{P}_{\text {start }}^{(m)}\left(\boldsymbol{x}^{(m)}\right) \mathcal{P}_{j}^{(s)}\left(\boldsymbol{x}^{(s)}\right) \mathcal{P}_{\text {blank }}^{(t)}\left(\boldsymbol{x}^{(t)}\right) .
$$

The distributions of the final states are denoted by $\mathcal{Q}_{j}$. Creating an exact copy of the source into the target implies that, for instance, the marginal distributions (of the final states) become

$$
\int d \boldsymbol{x}^{(m)} \mathcal{Q}_{j}=\mathcal{P}_{j}^{(s)}\left(\boldsymbol{x}^{(s)}\right) \mathcal{P}_{j}^{(s)}\left(\boldsymbol{x}^{(t)}\right) .
$$

It follows from (7) and (10), however, that the distance between the final states $\mathcal{Q}_{j}$ complies with the inequality

$$
K\left(\mathcal{Q}_{1}, \mathcal{Q}_{2}\right) \geq 2 K\left(\mathcal{P}_{1}^{(s)}, \mathcal{P}_{2}^{(s)}\right)=2 K\left(\mathcal{P}_{1}, \mathcal{P}_{2}\right) .
$$

If $K\left(\mathcal{P}_{1}^{(s)}, \mathcal{P}_{2}^{(s)}\right) \neq 0$, this inequality clearly conflicts with the conservation of the Kullback measure - see [19] for more details. Because the Kullback-Leibler distance requires that both of the distributions to be compared do not vanish everywhere as otherwise their logarithm tends to infinity, i.e., as the integral diverges, we further compare the distributions via the overlap function introduced earlier. Because of (8) and assuming that $\mathcal{P}_{\text {start }}^{(m)}$ and $\mathcal{P}_{\text {blank }}^{(t)}$ are normalized, the Kullback distance between the initial states (9) results in

$$
O\left(\mathcal{P}_{1}, \mathcal{P}_{2}\right)=O\left(\mathcal{P}_{1}^{(s)}, \mathcal{P}_{2}^{(s)}\right)
$$

Using the inequality of Schwarz we find

$$
\begin{aligned}
O\left(\mathcal{Q}_{1}, \mathcal{Q}_{2}\right)= & \int d \boldsymbol{x}^{(s)} d \boldsymbol{x}^{(t)} d \boldsymbol{x}^{(m)} \sqrt{\mathcal{Q}_{1} \mathcal{Q}_{2}} \\
\leq & \int d \boldsymbol{x}^{(s)} d \boldsymbol{x}^{(t)} \sqrt{\int d \boldsymbol{x}^{(m)} \mathcal{Q}_{1} \int d \boldsymbol{x}^{(m)} \mathcal{Q}_{2}} \\
\stackrel{(10)}{=} & \int d \boldsymbol{x}^{(s)} \sqrt{\mathcal{P}_{1}^{(s)}\left(\boldsymbol{x}^{(s)}\right) \mathcal{P}_{2}^{(s)}\left(\boldsymbol{x}^{(s)}\right)} \\
& \times \int d \boldsymbol{x}^{(t)} \sqrt{\mathcal{P}_{1}^{(s)}\left(\boldsymbol{x}^{(t)}\right) \mathcal{P}_{2}^{(s)}\left(\boldsymbol{x}^{(t)}\right)} \stackrel{(12)}{=}\left[O\left(\mathcal{P}_{1}, \mathcal{P}_{2}\right)\right]^{2} \\
\leq & O\left(\mathcal{P}_{1}, \mathcal{P}_{2}\right) .
\end{aligned}
$$

In (13) the equality requires that $\mathcal{Q}_{1} \propto Q_{2}$ and, consequently, $\mathcal{Q}_{1} \equiv \mathcal{Q}_{2}$, because $\mathcal{Q}_{j}$ are normalized. In sum, (11) and (13) form contradictions to the conservation of (5) and (6), respectively, provided that dynamical changes are given by the Liouville Eq. (2). Alternatively, we now consider initial states of the source system belonging to a family of probability distributions functions $\mathcal{P}(\boldsymbol{x} ; \lambda)$ characterized by a single parameter $\lambda$. The distance (3) between two close distributions belonging to this family is given as [31]

$$
G[\mathcal{P}(x ; \lambda), \mathcal{P}(x ; \lambda+\varepsilon)]=g(1)+C \varepsilon^{2} I[\mathcal{P}(x ; \lambda)]+\mathcal{O}\left(\varepsilon^{3}\right),
$$

where $C$ is a constant depending on the form of the function $g(\cdots)$. The form $I[\cdots]$ is Fisher's information measure, which reads

$$
I[\mathcal{P}(x ; \lambda)]=\int \frac{1}{\mathcal{P}}\left(\frac{\partial \mathcal{P}}{\partial \lambda}\right)^{2} d x .
$$

Fisher's information is a non-negative quantity that plays a key role in statistical estimation theory. Indeed, if one tries to infer the parameter $\lambda$ from one sample $x$ chosen from the distribution $\mathcal{P}$, then estimation theory asserts that the mean squared error $E^{2}$ for the (unbiased) estimation of $\lambda$ obeys the Cramer-Rao bound [4] 


$$
E^{2} \geq 1 / I[\mathcal{P}(\boldsymbol{x} ; \lambda)]
$$

in which equality is achieved for the "best" possible or efficient estimator. Coming back to the cloning problem, we assume that the initial states of the composite system involved in the process obey the form

$$
\mathcal{P}_{\lambda}=\mathcal{P}_{\text {start }}^{(m)}\left(\boldsymbol{x}^{(m)}\right) \mathcal{P}^{(s)}\left(\boldsymbol{x}^{(s)} ; \lambda\right) \mathcal{P}_{\text {blank }}^{(t)}\left(\boldsymbol{x}^{(t)}\right) .
$$

A successful cloning operation should yield final states

$$
\mathcal{Q}_{\lambda}=\mathcal{P}^{(m)}\left(\boldsymbol{x}^{(m)} ; \lambda\right) \mathcal{P}^{(s)}\left(\boldsymbol{x}^{(s)} ; \lambda\right) \mathcal{P}^{(s)}\left(\boldsymbol{x}^{(t)} ; \lambda\right) .
$$

Because Fisher's information is preserved under Liouville evolution, we have

$$
I\left[\mathcal{P}^{(s)}(\lambda)\right]=2 I\left[\mathcal{P}^{(s)}(\lambda)\right]+I\left[\mathcal{P}^{(m)}(\lambda)\right],
$$

which is clearly impossible provided $I\left[\mathcal{P}^{(s)}(\lambda)\right] \neq 0$. Interestingly, this result can be reinterpreted in terms of statistical estimation theory: we compare the Fisher measures associated with the initial state (17) and the final state (18), respectively, and obtain

$$
I\left[\mathcal{Q}_{\lambda}\right] \geq 2 I\left[\mathcal{P}_{\lambda}\right]
$$

if the transformation from (17) to (18) were possible, then we would be able to use the final state $\mathcal{Q}_{\lambda}$ to estimate the parameter $\lambda$ with an optimum mean squared error (assuming an efficient estimator)

$$
E^{2}\left[\mathcal{Q}_{\lambda}\right]=\frac{1}{2 I\left[\mathcal{P}_{\lambda}\right]+I\left[\mathcal{P}^{(m)}(\lambda)\right]}<\frac{1}{I\left[\mathcal{P}_{\lambda}\right]} .
$$

This form contradicts the Cramer-Rao inequality associated with the initial states $\mathcal{P}_{\lambda}$, because (16) provides a bound for the mean squared error associated with every (unbiased) estimation procedure. Note that the conservation of Fisher's information implies that the "distinguishability" of phase space ensembles does not change under Liouvillian evolution. On the contrary, final states generated by a universal cloning machine would be more "distinguishable" than the concomitant initial states.

Next to the no-cloning theorem, the so-called nondeleting theorem has a classical counterpart. To show this, we assume that the initial states of both the source and the target systems are described by the same probability distribution. Hence, the corresponding initial joint distribution of the tripartite system reads

$$
\mathcal{P}_{j}=\mathcal{P}_{\text {start }}^{(m)}\left(\boldsymbol{x}^{(m)}\right) \mathcal{P}_{j}^{(s)}\left(\boldsymbol{x}^{(s)}\right) \mathcal{P}_{j}^{(s)}\left(\boldsymbol{x}^{(t)}\right) .
$$

The aim of the process is to delete information of the target system against that of the source system, bringing the probability distribution of the former to a blank state so that final joint distribution becomes

$$
\mathcal{Q}_{j}=\mathcal{P}_{j}^{(m)}\left(\boldsymbol{x}^{(m)}\right) \mathcal{P}_{j}^{(s)}\left(\boldsymbol{x}^{(s)}\right) \mathcal{P}_{\text {blank }}\left(\boldsymbol{x}^{(t)}\right) .
$$

The conservation of the Kullback distance provides

$$
K\left(\mathcal{P}_{1}^{(s)}, \mathcal{P}_{2}^{(s)}\right)=K\left(\mathcal{P}_{1}^{(m)}, \mathcal{P}_{2}^{(m)}\right),
$$

implying that the information deleted from the target system is entirely transferred into the final state of the deleting machine. Similarly, by assuming $O\left(\mathcal{P}_{1}^{(s)}, \mathcal{P}_{2}^{(s)}\right) \neq$ 0 , the conservation of the overlap yields

$$
O\left(\mathcal{P}_{1}^{(s)}, \mathcal{P}_{2}^{(s)}\right)=O\left(\mathcal{P}_{1}^{(m)}, \mathcal{P}_{2}^{(m)}\right) .
$$

Again, all the information distance between the states to be deleted is transferred into the final states of the deleting machine.

In line with [19], we extend the present discussion to the case in which the initial state of the source-target system cannot be factorized. For this sake, we consider an initial state

$$
\begin{aligned}
\mathcal{P}_{j} & =\mathcal{P}_{\text {start }}^{(m)}\left(\boldsymbol{x}^{(m)}\right) \mathcal{P}_{j}^{(s, t)}\left(\boldsymbol{x}^{(s)}, \boldsymbol{x}^{(t)}\right) \\
& =\mathcal{P}_{\text {start }}^{(m)}\left(\boldsymbol{x}^{(m)}\right) \mathcal{P}_{j}^{(s)}\left(\boldsymbol{x}^{(s)}\right) h\left(\boldsymbol{x}^{(s)}, \boldsymbol{x}^{(t)}\right),
\end{aligned}
$$

where $\mathcal{P}_{j}^{(s, t)}\left(\boldsymbol{x}^{(s)}, \boldsymbol{x}^{(t)}\right)$ and $h\left(\boldsymbol{x}^{(s)}, \boldsymbol{x}^{(t)}\right)$ are symmetrical functions of their respective arguments. $\mathcal{P}_{j}^{(s)}\left(\boldsymbol{x}^{(s)}\right)=$ $\int \mathcal{P}_{j}^{(s, t)}\left(\boldsymbol{x}^{(s)}, \boldsymbol{x}^{(t)}\right) d \boldsymbol{x}^{(t)}$ is the marginal probability distribution for $\boldsymbol{x}^{(s)}$ and $h\left(\boldsymbol{x}^{(s)}, \boldsymbol{x}^{(t)}\right)$ denotes the conditional probability distribution of $\boldsymbol{x}^{(t)}$ for a given value of $\boldsymbol{x}^{(s)}$ (irrespective of $j$ ). Given these specific assumptions, the transformation from the initial state (26) into a final state $\mathcal{P}_{\text {final }}^{(m)}\left(\boldsymbol{x}^{(m)}\right) \mathcal{P}_{j}^{(s)}\left(\boldsymbol{x}^{(s)}\right) \mathcal{P}_{\text {blank }}\left(\boldsymbol{x}^{(t)}\right)$ is not in conflict with the conservation of the Kullback distance. This result is consistent with Landauer's assertion that, in classical systems, it is possible to erase a bit against its copy. A physical scenario corresponding to the erasure of 1 bit against its copy can be represented by an initial ensemble of bipartite source-target systems, such that in each one the source and the target are in the same state (representing a bit and its copy). This ensemble is described by a distribution of the form (26) with $h\left(\boldsymbol{x}^{(s)}, \boldsymbol{x}^{(t)}\right)=$ $\delta\left(\boldsymbol{x}^{(s)}-\boldsymbol{x}^{(t)}\right)$.

To briefly discuss the deleting process in terms of Fisher's measure, we consider initial states $\mathcal{P}_{\lambda}=$ $\mathcal{P}_{\text {start }}^{(m)}\left(\boldsymbol{x}^{(m)}\right) \mathcal{P}^{(s)}\left(\boldsymbol{x}^{(s)} ; \lambda\right) \mathcal{P}^{(s)}\left(\boldsymbol{x}^{(t)} ; \lambda\right)$ being defined in terms of the mono parametric family of distributions $\mathcal{P}^{(s)}\left(\boldsymbol{x}^{(s)} ; \lambda\right)$. A universal deleting process would lead to final states of the form $\mathcal{Q}_{\lambda}=\mathcal{P}^{(m)}\left(\boldsymbol{x}^{(m)} ; \lambda\right) \times$ $\mathcal{P}^{(s)}\left(\boldsymbol{x}^{(s)} ; \lambda\right) \mathcal{P}_{\text {blank }}\left(\boldsymbol{x}^{(t)}\right)$. The conservation of Fisher's information, however, implies $I\left[\mathcal{P}^{(s)}(\lambda)\right]=I\left[\mathcal{P}^{(m)}(\lambda)\right]$. That is, the Fisher information associated with the initial states of the target systems is entirely transferred to the final states of the machine. One may argue that the distinguishability of the initial target states (which is lost during the deleting process) is completely transformed into an equal amount of distinguishability of the final state of the machine.

As indicated above, even if Liouville dynamics forbids universal cloning or deleting of ensemble distributions, the cloning or deleting of some particular distributions are not necessarily forbidden. For instance, if the states to 
be cloned or deleted are "nonoverlapping," the Kullback distance is not defined and our present arguments do not hold. Further, in this case the overlap distance between two initial states of the cloning process is equal to the distance between the corresponding final states: both distances vanish. Hence, the conservation of distance is not violated. Similarly, the overlap distances between initial states and between final states of a successful deleting process involving two nonoverlapping distributions also vanish. Consequently, the distance can be preserved without transferring any information into the final states of the deleting machine. Entirely known classical states described by $\delta$ distributions are special instances of this nonoverlapping situation.

Information distances between time-dependent solutions of the Liouville equation constitute integrals of motion of the concomitant dynamics. The invariance of these measures imposes rather strong constraints on possible universal operations in classical ensemble dynamics. These constraints allow for the identification of classical analogues of information-related, quantum mechanical impossible operations such as universal quantum cloning and universal quantum deleting. The Fisher information measure provides an interesting interpretation of these classically forbidden operations in terms of statistical inference theory. The physical impossibility of universal cloning or deleting is a basic feature of classical probabilistic settings arising from an incomplete knowledge of the system's state. However, complete knowledge of classical states is possible, at least in principle, and cloning and deleting are not forbidden in such cases (they are possible even in the more general case of nonoverlapping probability distributions). In this regard, the quantum mechanical situation is more strict since universal cloning or deleting are impossible even within the set of completely determined states, that is, for pure states [20-22].

The present formalism may be applied to investigate classical counterparts of other quantum impossible processes. Likewise, possible links between our results and the classical analogue of entanglement analyzed in [15] deserve in-depth study.

We thank the University of Pretoria for financial support. A. D. is grateful for the hospitality of the Physics Department of the University of Pretoria.

*Electronic address: arplastino@maple.up.ac.za

†Electronic address: marlow@fbw.vu.nl

[1] Complexity, Entropy, and the Physics of Information edited by W. H. Zurek (Addison- Wesley, Redwood City, California, 1990).

[2] C. Beck and F. Schlogl, Thermodynamics of Chaotic Systems (Cambridge University Press, Cambridge, 1993).
[3] J. D. Barrow and F. J. Tipler, The Antropic Cosmological Principle (Oxford University Press, Oxrord, 1996).

[4] B. R. Frieden, Physics from Fisher Information (Cambridge University Press, Cambridge, 1998).

[5] B. R. Frieden, Science from Fisher Information (Cambridge University Press, Cambridge, 2004).

[6] B. R. Frieden and B. H. Soffer, Phys. Rev. E 52, 2274 (1995).

[7] M. J.W. Hall, Phys. Rev. A 59, 2602 (1999).

[8] B. Piechocinska, Phys. Rev. A 61, 062314 (2000).

[9] P.T. Landsberg, Seeking Ultimates (Institute of Physics Publishing, Bristol and Philadelphia, 2001).

[10] H. C. von Baeyer, Information: The New Language of Science (Weidenfeld and Nicolson, London, 2003).

[11] J. Gemmer, A. Otte, and G. Mahler, Phys. Rev. Lett. 86, 1927 (2001).

[12] A. Hosoya, T. Buchert, and M. Morita, Phys. Rev. Lett. 92, 141302 (2004).

[13] M. Nielsen and I. Chuang, Quantum Computation and Information (Cambridge University Press, Cambridge, 2000).

[14] Maxwell's Demons 2: Entropy, Classical and Quantum Information, Computing edited by H.S. Leff and A. F. Rex (Institute of Physics Publishing, Bristol and Philadelphia, 2003).

[15] D. Collins and S. Popescu, Phys. Rev. A 65, 032321 (2002).

[16] L. K. Grover and A. M. Sengupta, Phys. Rev. A 65, 032319 (2002).

[17] M. D. Westmoreland and B.W. Schumacher, Phys. Rev. A 48, 977 (1993).

[18] M. D. Westmoreland, J. Krone, and B.W. Schumacher, Physica D 120, 236 (1998).

[19] A. Daffertshofer, A. R. Plastino, and A. Plastino, Phys. Rev. Lett. 88, 210601 (2002).

[20] W. K. Wootters and W. H. Zurek, Nature 299, 802 (1982).

[21] D. Dieks, Phys. Lett. A 92, 271 (1982).

[22] A. K. Pati and S. L. Braunstein, Nature (London) 404, 164 (2000).

[23] T. Mor, Phys. Rev. Lett. 83, 1451 (1999).

[24] A. K. Pati, Phys. Rev. A 66, 062319 (2002).

[25] A. K. Pati and S. L. Braunstein, Phys. Lett. A 315, 208 (2003).

[26] C. H. Bennett, G. Brassard, and N. D. Mermin, Phys. Rev. Lett. 68, 557 (1992).

[27] N. Gisin and S. Massar, Phys. Rev. Lett. 79, 2153 (1997).

[28] T. M. Cover and J. A. Thomas, Elements of Information Theory (Wiley-Interscience, New York, 1991).

[29] We are not assuming Hamiltonian dynamics, which is a particular instance of (1). For a Hamiltonian system with $n$ degrees of freedom we have $N=2 n, \quad \mathbf{x}=$ $\left(q_{1}, \ldots, q_{n}, p_{1}, \ldots, p_{n}\right), v_{i}=\partial H / \partial p_{i}(i=1, \ldots, n)$, and $v_{i}=-\partial H / \partial q_{i}(i=n+1, \ldots, 2 n)$; the $q_{i}$ and $p_{i}$ stand for generalized coordinates and momenta, respectively.

[30] W. K. Wootters, Phys. Rev. D 23, 357 (1981).

[31] L. Borland, A. R. Plastino, and C. Tsallis, J. Math. Phys. (N.Y.) 39, 6490 (1998). 